\title{
Utility of Biomarkers in the Evaluation of Fever in the Intensive Care Unit After Brain Injury
}

\author{
Umer Mukhtar, MD; Umer Shoukat, MD; M. Kamran Athar, MD; \\ Fred Rincon, $M D^{1,2}$ \\ ${ }^{1}$ Department of Neurological Surgery, Thomas Jefferson University, Philadelphia, PA \\ 2 Department of Neurology, Thomas Jefferson University, Philadelphia, PA
}

\begin{abstract}
Fever is frequent in patients with neurologic injury. Differentiating infectious fever from central fever can be challenging. It is important to diagnose the cause of fever in the neurological intensive care unit because of the detrimental effects of fever on brain injured patients. This is a comprehensive review of the role of the two commonly available biomarkers, C-reactive protein and procalcitonin in differentiating the central fever from infectious fever.
\end{abstract}

Key words

Proclacitonin, C reactive protein, Subarachnoid hemorrhage, Intracerebral hemorrhage.

\section{INTRODUCTION AND BACKGROUND}

Fever is frequently seen in the neurologic intensive care unit (NICU). Incidence rates of up to $70 \%$ have been reported in various studies. ${ }^{1-5}$ Fever can help host defenses by local activation of the coagulation cascade, cytokine-mediated T-cell activation, as well as neutrophil and macrophage recruitment to injured tissues. In brain injured patients, after the initial insult, secondary neuronal injury is speculated to be caused by several processes including mitochondrial dysfunction, inflammatory response, free radical generation, and excitatory neurotransmitter release. Fever has been shown to exacerbate secondary neuronal injury and physiologic dysfunction after traumatic brain injury (TBI), subarachnoid hemorrhage (SAH), and major neurosurgery. ${ }^{6}$

\section{CAUSES OF FEVER IN NEUROLOGICALLY INJURED PATIENTS}

Infections are the most common cause of fever in the NICU population, accounting for at least half of the febrile episodes. ${ }^{2,4-7}$ A significant percentage of patients in the NICU have central fevers. Central fever results from loss of physiological regulation of body temperature by the hypothalamus. ${ }^{7}$ The diagnosis of central fever is challenging as there is no clear definition or diagnostic criteria. In addition, the prevalence of systemic inflammatory response syndrome (SIRS) and leukocytosis may be similar in patients with both central and infectious fevers. ${ }^{8}$ When there is clinical suspicion of infection, it is common practice to initiate broad spectrum antibiotics after obtaining appropriate culture specimens in ICU patients. This practice results in another challenge for neurointensivists as many patients with brain injury have central fever and antibiotics may be continued unnecessarily in these patients. This may result in the emergence of antibiotic resistant organisms, increase side effects of antibiotics as well as healthcare costs. A better understanding of predictors of central fever is important as it will help in antibiotic stewardship and may also allow for earlier discontinuation of antibiotics in patients with central fever. ${ }^{2}$ Rabinstein et al identified a number of variables that are predictive of central fever in the neurocritical care population. Their study showed that in patients with onset of fever within 72 hours of admission, patients with persistent fevers, negative cultures and lack of infiltrates on chest X-ray were more likely to have central fever. This was especially true if their primary diagnosis was
$\mathrm{SAH}$, tumor or they had intraventricular hemorrhage. ${ }^{8}$

In addition to these variables, a biomarker predictive of sepsis can be helpful in differentiating infectious from central fever and can also aid in antibiotic stewardship.

\section{Overview of Biomarkers}

Ideally, in order to guide antibiotic use, clinicians need a valid, reliable and readily available test that would correlate well with their clinical suspicion and also help them in differentiating between central and infectious fever. A number of biomarkers have been studied in patients with sepsis, some of which have also been evaluated in brain injured patients. One of the more promising ones is procalcitonin (PCT). Other biomarkers include $C$ reactive protein (CRP) and Interleukin-6 (IL-6). These biomarkers and their validity, efficacy and availability in intensive care unit are discussed under.

\section{Procalcitonin}

Procalcitonin (PCT) is a glycopeptide consisting of 116 amino acids produced under normal conditions in the $C$ cells of the thyroid gland as the precursor molecule of calcitonin. Several studies have demonstrated that PCT levels are raised in severe invasive bacterial infections and decrease rapidly after appropriate antibiotic therapy. ${ }^{9-14}$ In contrast, PCT levels are normal or only slightly increased in localized bacterial infections, viral infections, and inflammatory reactions of noninfectious origin. 9,15-17 There is recent evidence suggesting that PCT can distinguish sepsis from non-infectious SIRS in general critical care patients, allowing clinicians to make better diagnostic and therapeutic decisions. ${ }^{18-21}$

\section{Procalcitonin vs. Other Biomarkers}

A number of studies have evaluated the role of PCT in comparison with CRP and IL- 6 as a diagnostic and prognostic 
biomarker. In a prospective cohort study Ville Pettilä et al evaluated the predictive value of PCT and IL- 6 in patients with suspected sepsis. PCT and IL- 6 values on day 2 of suspected sepsis were independently predictive of hospital mortality. ${ }^{22}$

Simon et al conducted a systemic review and meta-analysis. They studied the relation between serum PCT and CRP levels as a marker of inflammation. PCT had higher accuracy than CRP for discriminating bacterial infections from non-infectious causes of inflammation. In addition PCT was also significantly better than CRP in differentiating bacterial from viral infections. ${ }^{23}$

Choi et al conducted a prospective study to evaluate the predictive performance of serum PCT as a differentiating marker between postoperative bacterial meningitis (PBM) and postoperative aseptic meningitis (PAM). For the diagnosis of PBM, PCT level $\geq 0.15 \mathrm{ng} / \mathrm{mL}$ had a specificity of $80.0 \%$ and sensitivity of $50 \%$. The combined criteria of a CRP level $\geq 2.5 \mathrm{mg} / \mathrm{dL}$, WBC count $\geq 9,500 / \mathrm{mm} 3$, and PCT level $\geq 0.15 \mathrm{ng} / \mathrm{mL}$ had the highest specificity of $92.6 \%$ and higher sensitivity of $85.7 \%$. They concluded that PCT alone has a limited performance for the diagnosis of PBM, but has improved diagnostic value when used as an adjunct test with other inflammatory markers. ${ }^{24}$

\section{Procalcitonin as a biomarker for diagnosis of sepsis in brain injured patients}

Fever is common in brain injured patients. As stated earlier, a significant proportion of these patients have central fever and differentiating central from infectious fever is challenging. In a prospective observational study, Festic et al showed that PCT may be a useful tool when managing SIRS in a patient with aneurysmal subarachnoid hemorrhage (aSAH). They found that in these patients, serum PCT values have high specificity, high negative predictive value and good overall predictive utility for infections, particularly for major infections. Patients with infection were $>25$ times more likely to have an elevated PCT values compared to those with no infection. For patients with a major infection, the odds ratio was even higher (>33). ${ }^{25}$
Early diagnosis of bacterial or viral meningitis is important so that antibiotic treatment can be started without delay. For immediate diagnosis of bacterial meningitis, the sensitivity of direct cerebrospinal fluid (CSF) examination or the detection of bacterial antigens in CSF is low. ${ }^{26-29}$

Viallon et al conducted a prospective study to determine the ability of inflammatory biomarkers commonly used for the diagnosis of acute meningitis to differentiate between bacterial and viral meningitis, in adult patients with a negative CSF examination. Out of 254 patients with meningitis with a negative direct CSF examination, 35 had bacterial meningitis and 181 had viral meningitis. Serum PCT was a highly discriminative biomarker and had a sensitivity of $95 \%$, a specificity of $100 \%$, a negative predictive value of $100 \%$, and a positive predictive value of $97 \%$ at a diagnostic cut-off level of $0.28 \mathrm{ng} / \mathrm{ml}$. 30

In another prospective study, Berger et al measured daily PCT levels in patients requiring temporary external ventricular drains (EVD). They showed that PCT levels were significantly higher (4.7 vs 0.2 $\mathrm{ng} / \mathrm{ml}$ ) in patients with proven cerebral ventriculitits. CSF cell count could not differentiate bacterial infections from abacterial reactions. ${ }^{31}$

Martinez et al measured serum PCT in 15 consecutive patients with ventriculitis

\section{Figure 1. Studies describing the role of Procalcitonin as a differentiating marker.}

\begin{tabular}{|l|l|l|l|l|l|l|}
\hline Study & PCT Cut off & $\begin{array}{l}\text { No. of } \\
\text { Patients }\end{array}$ & Specificity & Sensitivity & PPV & NPV \\
\hline $\begin{array}{l}\text { Festic et al. } \\
\text { (25) }\end{array}$ & 0.2 & 40 & 97.7 & 37.5 & 85.7 & 80.8 \\
\hline $\begin{array}{l}\text { Viallon et al. } \\
\text { (29) }\end{array}$ & 0.28 & 254 & 100 & 95 & 97 & 100 \\
\hline $\begin{array}{l}\text { Berger et al. } \\
\text { (31) }\end{array}$ & $>1$ & 34 & 100 & 100 & 100 & 100 \\
\hline $\begin{array}{l}\text { Schwarz et al. } \\
\text { (33) }\end{array}$ & $>0.5$ & 30 & 100 & 69 & 100 & 74 \\
\hline $\begin{array}{l}\text { Martinez et al. } \\
\text { (32) }\end{array}$ & 1 & 15 & 77 & 68 & NA & NA \\
\hline
\end{tabular}

PCT: Procalcitonin. PPV: Positive Predictive Value. NPV: Negative Predictive Value.

PCT Cut of value is $\mathrm{ng} / \mathrm{ml}$ for all studies. in which an EVD had been inserted and compared the data with ten patients who had bacterial meningitis. Four out of fifteen patients had microbiologically proven bacterial ventriculitis with positive bacterial cultures. PCT value of $1.0 \mathrm{ng} / \mathrm{ml}$ showed a specificity of $77 \%$ and sensitivity of $68 \%$ in patients with ventriculitis with positive CSF bacterial cultures. ${ }^{32}$

In a prospective case series Schwarz et al compared serum PCT levels in patients with bacterial meningitis to those with abacterial meningitis. At admission, PCT levels were significantly higher in patients with bacterial meningitis as compared with those with abacterial meningitis ( $p$ $<.001$ ). The specificity of PCT was $100 \%$ for bacterial infections, but there were false-negative findings in five patients with bacterial meningitis (a sensitivity of $69 \%$ ). Persistently elevated or increasing PCT levels after 2 days were associated with an unfavorable clinical course. ${ }^{33}$

In a case series comparing 7 patients with Neuro-Behcet disease to 3 patients with bacterial meningitis, Suzuki et al showed that serum PCT levels were increased in patients with bacterial meningitis, but not in those with NeuroBehcet disease. Therefore, serum PCT may be a useful marker for discrimination between Neuro-Behcet disease and septic meningitis, especially in cases of the meningeal form of Neuro-Behcet disease. ${ }^{34}$ 
Results of these studies show that PCT can serve as a valuable tool in differentiating central fever from infectious fever, as well as discriminating primary bacterial CNS infections from non-bacterial CNS inflammatory processes.

\section{C-Reactive Protein as a biomarker in Neurologically Injured Patients:}

CRP is a major acute-phase plasma protein which is rapidly released in response to infection or tissue injury. We will discuss the role of CRP as a differentiating marker in CNS infections and other CNS inflammatory conditions. In a retrospective analysis of 35 patients with brain abscess requiring surgical drainage, Neidert et al found that preoperative mean CRP levels were significantly higher in the group requiring repeated surgical drainage. They concluded that patients with higher CRP level at baseline should be monitored closely to determine the need for repeat surgical drainage. ${ }^{35}$

Cerebral vasospasm is a common and potentially devastating complication of aneurysmal subarachnoid hemorrhage (aSAH). An inflammatory mechanism is implicated in the development of vasospasm. In a prospective study of 61 adult patients with aSAH, Hwang et al measured Serum CRP levels on days 1, 3, $5,7,9,11$ and 13 after aneurysm rupture. They found that Serum CRP levels peaked on the 3rd postoperative day and there were significant differences between the vasospasm group and the nonvasospasm group on the days 1, 3 and 5 . They suggested that these patients may require closer observation to monitor for the development of vasospasm. ${ }^{36}$

In a prospective study of 100 adult patients with aneurysmal SAH, Romero et al showed that higher serum CRP levels are associated with worse clinical outcome and the occurrence of delayed ischemic neurological deficits. ${ }^{37}$ In a prospective study, Napoli et al evaluated whether elevation of white blood cell count (WBC), C-reactive protein (CRP), and blood glucose (BG) concentration at presentation were prognostic of poor outcome in spontaneous Intracerebral Hemorrhage $(\mathrm{s} I \mathrm{CH})$ patients. Higher WBC, CRP, and BG were associated with increased mortality in univariate analysis.
However, only CRP elevation remained associated with mortality in the multivariate model, after adjusting for multiple confounders. ${ }^{38}$

The review of the studies related to CRP show that it is raised universally in all kinds of inflammatory responses, whether infectious or noninfectious. It may be useful in addition to PCT, but it cannot be solely used to differentiate between infectious and noninfectious fever. As a result its utility for antibiotic stewardship in NICU is limited.

\section{CONCLUSIONS AND FUTURE APPROACHES:}

The development of fever in critically ill patient needs immediate attention and action to rule out infection. This situation is more challenging in the NICU patient population due to the high rate of noninfectious fever. On one hand there is high morbidity and mortality associated with sepsis, but on the other hand use of broad spectrum antibiotics in patients who do not have infection results in high rates of antibiotic resistance, infections, and adverse drug reactions. In order to help diagnosis, a test is definitely needed that can differentiate between infectious and noninfectious fever. If a test helps us rule out infection and diagnose central fever correctly, then in spite of unnecessary use of antibiotics, we can use novel techniques such as surface or intravascular cooling measures like artic sun to treat hyperthermia in brain injured patients.

We conclude from the above review of literature that PCT may be a more sensitive and specific test that can help us differentiate between infectious and central fever. In addition it can also be a useful test to differentiate between primary bacterial CNS infections vs other types of CNS infections or non-infectious CNS inflammatory processes. However, data is very limited in neurocritical and neurosurgical patient populations. More clinical studies and clinical trials are needed that can validate the use of PCT as a diagnostic test to differentiate between infectious and noninfectious fever. In addition, the diagnostic cut-off levels of PCT also need to be validated.

\section{REFERENCES}

1. Occurrence of potentially detrimental temperature alterations in hospitalized patients at risk for brain injury. Albrecht RF 2nd, et al. Mayo Clin Proc. 1998 Jul;73(7):629-35.

2. Hyperthermia in the neurosurgical intensive care unit. Kilpatrick MM, et al. Neurosurgery. 2000 Oct;47(4):850-5.

3. Pyrexia in head-injured patients admitted to intensive care. Stocchetti N, et al. Intensive Care Med. 2002 Nov;28(11):1555-62.

4. Risk factors for fever in the neurologic intensive care unit. Commichau $C$, et al. Neurology. 2003 Mar 11;60(5):837-41.

5. Elevated body temperature independently contributes to increased length of stay in neurologic intensive care unit patients. Diringer MN, et al. Crit Care Med. 2004 Jul;32(7):1489-95.

6. Patterns of increased intracranial pressure after severe traumatic brain injury. O'Phelan KH, et al. Neurocrit Care. 2009;10(3):280-6. doi: 10.1007/s12028-008-9183-7.

7. Non-infectious fever in the neurological intensive care unit: incidence, causes and predictors. Rabinstein AA, et al. J Neurol Neurosurg Psychiatry. 2007 Nov; $78(11): 1278-80$.

8. Indicators of central fever in the neurologic intensive care unit. Hocker SE, et al. JAMA Neurol. 2013 Dec;70(12):1499-504.

9. High serum procalcitonin concentrations in patients with sepsis and infection. Assicot M, et al. Lancet. 1993 Feb 27;341(8844):515-8.

10. Elevated serum procalcitonin levels in patients with melioidosis. Smith MD, , et al. Clin Infect Dis. 1995 Mar;20(3):641-5.

11. Procalcitonin as a marker for the early diagnosis of neonatal infection. Gendrel D, et al. J Pediatr. 1996 Apr;128(4):570-3.

12. Procalcitonin in patients with and without immunosuppression and sepsis. al-Nawas B, et al. Infection. 1996 Nov-Dec;24(6):434-6

13. Reliability of procalcitonin concentrations for the diagnosis of sepsis in critically ill neonates. Chiesa C, et al. Clin Infect Dis. 1998 Mar;26(3):664-72.

14. Procalcitonin and $C$-reactive protein levels in neonatal infections. Monneret G, et al. Acta Paediatr. 1997 Feb;86(2):209-12

15. Usefulness of procalcitonin for differentiation between activity of systemic autoimmune disease (systemic lupus erythematosus/ systemic antineutrophil cytoplasmic antibodyassociated vasculitis) and invasive bacterial infection. Eberhard OK, et al. Arthritis Rheum. 1997 Jul;40(7):1250-6.

16. The potential role of procalcitonin and interleukin 8 in the prediction of infected necrosis in acute pancreatitis. Rau B, et al. Gut. 1997 Dec; 41(6):832-40. 
17. Cytokines, nitrite/nitrate, soluble tumor necrosis factor receptors, and procalcitonin concentrations: comparisons in patients with septic shock, cardiogenic shock, and bacterial pneumonia. de Werra I, et al. Crit Care Med. 1997 Apr;25(4):607-13.

18. Efficacy of procalcitonin in the early diagnosis of bacterial infections in a critical care unit. Nakamura A, et al. Shock. 2009 Jun;31(6):586-91

19. Diagnostic value of procalcitonin, interleukin-6, and interleukin- 8 in critically ill patients admitted with suspected sepsis. Harbarth S, et al. Am J Respir Crit Care Med. 2001 Aug 1;164(3):396-402

20. Calcitonin precursors are reliable markers of sepsis in a medical intensive care unit. Müller B, et al. Crit Care Med. 2000 Apr;28(4):977-83.

21. Use of procalcitonin for early prediction of lethal outcome of postoperative sepsis. Novotny A, et al. Am J Surg. 2007 Jul;194(1):35-9.

22. Predictive value of procalcitonin and interleukin 6 in critically ill patients with suspected sepsis. Pettilä V, et al. Intensive Care Med. 2002 Sep;28(9):1220-5

23. Serum procalcitonin and C-reactive protein levels as markers of bacterial infection: a systematic review and meta-analysis. Simon L, et al. Clin Infect Dis. 2004 Jul 15;39(2):206-17.
24. Predictive performance of serum procalcitonin for the diagnosis of bacterial meningitis after neurosurgery. Choi $\mathrm{SH}_{\text {, Infect }}$ Chemother. 2013 Sep;45(3):308-14.

25 . The utility of serum procalcitonin in distinguishing systemic inflammatory response syndrome from infection after aneurysmal subarachnoid hemorrhage. Festic $E$, et al. Neurocrit Care. 2014 Jun;20(3):375-81.

26. The spinal tap: a new look at an old test. Marton K, et al. Ann Intern Med. 1986 Jun;104(6):840-8.

27. Clinical features and prognostic factors in adults with bacterial meningitis. van de Beek D, et al. N Engl J Med. 2004 Oct 28;351(18):1849-59.

28. Differential diagnosis of acute meningitis An analysis of the predictive value of initial observations. Spanos A, et al. JAMA. 1989 Nov 17:262(19):2700-7.

29. High sensitivity and specificity of serum procalcitonin levels in adults with bacterial meningitis. Viallon A, et al. Clin Infect Dis. 1999 Jun;28(6):1313-6.

30. Meningitis in adult patients with a negative direct cerebrospinal fluid examination: value of cytochemical markers for differential diagnosis. Viallon A, et al. Crit Care 2011;15(3):R136
31. Serum procalcitonin in cerebral ventriculitis. Berger C, et al. Crit Care Med. 2002 Aug;30(8):1778-81

32. Serum procalcitonin monitoring for differential diagnosis of ventriculitis in adult intensive care patients. Martínez R, et al. Intensive Care Med. 2002 Feb;28(2):208-10.

33. Serum procalcitonin levels in bacterial and abacterial meningitis. Schwarz S, et al. Crit Care Med. 2000 Jun;28(6):1828-32.

34. Procalcitonin might help in discrimination between meningeal neuro-Behçet disease and bacterial meningitis. Suzuki $\mathrm{N}$, et al. Neurology. 2009 Feb 24;72(8):762-3.

35. Preoperative $\mathrm{C}$-reactive protein predicts the need for repeated intracerebral brain abscess drainage. Neidert MC, et al. Clin Neurol Neurosurg. 2015 Apr;131:26-30

36. Significance of C-reactive protein and transcranial Doppler in cerebral vasospasm following aneurysmal subarachnoid hemorrhage. Hwang $\mathrm{SH}$, et al. J Korean Neurosurg Soc. 2013 Oct; 54(4):289-95.

37. C-reactive protein and vasospasm after aneurysmal subarachnoid hemorrhage. Romero FR, et al. Acta Cir Bras. 2014 May;29(5):340-5.

38. C-reactive protein level measurement improves mortality prediction when added to the spontaneous intracerebral hemorrhage score. Di Napoli M, et al. Stroke. 2011 May;42(5):1230-6.

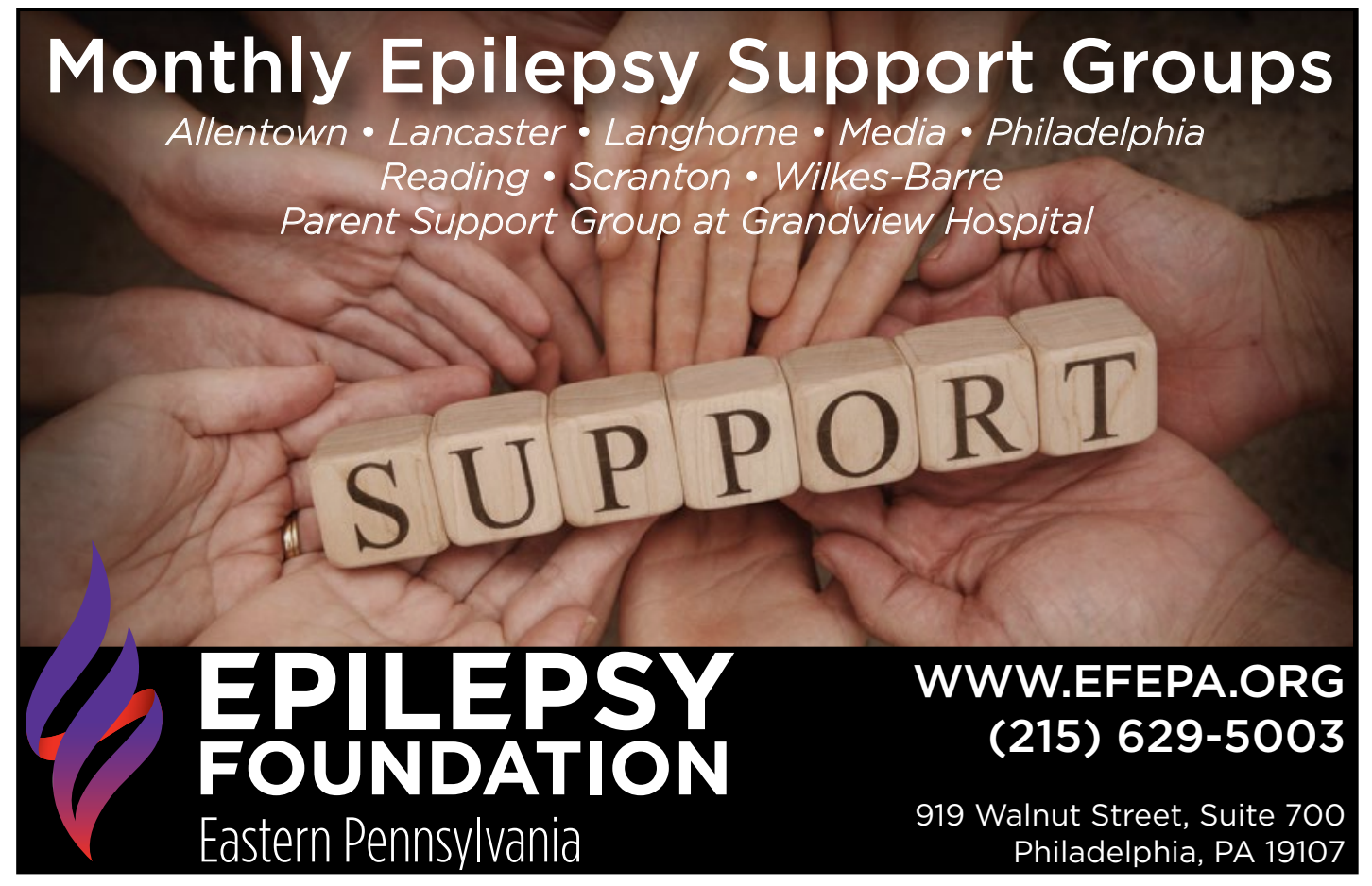

